\title{
THE STABILITY OF PURE WEIGHTS UNDER CONDITIONING
}

\author{
by D. J. FOULIS and C. H. RANDALL
}

(Received 27 June, 1972)

1. Introduction. In [1], we showed how a collection of physical operations or experiments could be represented by a nonempty set $\mathscr{A}$ of nonempty sets satisfying certain conditions (irredundancy and coherence) and we called such sets $\mathscr{A}$ manuals. We also introduced " complete stochastic models" for the empirical universe of discourse represented by such a manual $\mathscr{A}$, namely, the so-called weight functions for $\mathscr{A}$. These weight functions form a convex set the extreme points of which are called pure weights. We also showed that there is a so-called logic $\Pi(\mathscr{A})$ affiliated with a manual $\mathscr{A}$ and that each weight function for $\mathscr{A}$ induces a state on this logic.

In practice, physical operations are usually synthesized from " simpler" or more " primitive" operations by iteration or compounding. In [8], we gave an indication of a mathematical construction whereby such compound operations can be given a perspicuous representation. Specifically, given a manual $\mathscr{A}$, one can construct from it a new manual $\mathscr{A}^{c}$ whose elements represent compound operations built up from the operations in the parent manual $\mathscr{A}$. In [9], we gave an indication of how the weight functions on the parent manual $\mathscr{A}$ induce (by means of so-called transition functions) weight functions on the compound manual $\mathscr{A}^{c}$.

We showed in [2] that the weight functions for the compound manual $\mathscr{A}^{c}$ can be transformed by certain natural conditioning maps into new weight functions for $\mathscr{A}^{c}$. In the present paper, we shall concern ourselves with the investigation of the stability of pure weight functions for $\mathscr{A}^{c}$ under these conditioning maps. It will be convenient to deal with premanuals, which are generalized manuals, rather than with manuals. Premanuals, which will shortly be defined, were first studied (under a different name) by Greechie and Miller in [4].

2. Premanuals and weight functions. By a premanual we mean a nonempty set $\mathscr{A}$ of nonempty sets. If $\mathscr{A}$ is such a premanual, the set $X=\mathrm{U} \mathscr{A}$ is called the set of outcomes of $\mathscr{A}$. By a weight function for the premanual $\mathscr{A}$ we mean a real valued function $\omega$ defined on the outcome set $X=\bigcup \mathscr{A}$ and satisfying the following two conditions: (i) $0 \leqq \omega(x) \leqq 1$ for all $x \in X$. (ii) For each $E \in \mathscr{A}$, the unordered sum $\sum_{x \in E} \omega(x)$ converges to 1. In [3], Greechie has given examples of premanuals affiliated with finite orthomodular lattices which admit no weight functions whatsoever. We shall denote the set of all weight functions for the premanual $\mathscr{A}$ by $\Omega(\mathscr{A})$.

If $\mathscr{A}$ is a premanual and if $\alpha, \beta \in \Omega(\mathscr{A})$, we define, for each real number $t$, a mapping $t \alpha+(1-t) \beta$ from $X=\bigcup \mathscr{A}$ to the real numbers by

$$
(t \alpha+(1-t) \beta)(x)=t \alpha(x)+(1-t) \beta(x)
$$

for all $x \in X$. Evidently, if $0 \leqq t \leqq 1$, then $t \alpha+(1-t) \beta \in \Omega(\mathscr{A})$; hence, in this case, we refer to $t \alpha+(1-t) \beta$ as a convex combination of the weight functions $\alpha$ and $\beta$. A weight function 
$\omega \in \Omega(\mathscr{A})$ is said to be pure if it cannot be written, nontrivially, as a convex combination of weight functions $\alpha$ and $\beta$. Specifically, $\omega$ is a pure weight function if and only if $\omega=t \alpha+(1-t) \beta$ with $\alpha, \beta \in \Omega(\mathscr{A})$ and $0<t<1$ implies that $\alpha=\beta$. We denote by $\Omega_{p}(\mathscr{A})$ the set of all pure weight functions for $\mathscr{A}$.

If $\mathscr{A}$ is a premanual and if $\alpha, \beta \in \Omega(\mathscr{A})$, we define a real number $r(\alpha, \beta)$ by the following:

$$
r(\alpha, \beta)=\inf \left\{\frac{\alpha(x)}{\beta(x)} \mid x \in X=\bigcup \mathscr{A} \text { and } \beta(x) \neq 0\right\} \text {. }
$$

Evidently, $0 \leqq r(\alpha, \beta)$. If $1 \leqq r(\alpha, \beta)$, then $\beta(x) \leqq \alpha(x)$ for all $x \in X=\bigcup \mathscr{A}$, from which it easily follows that $\beta=\alpha$ and $r(\alpha, \beta)=1$. In particular, then, if $\alpha \neq \beta, 0 \leqq r(\alpha, \beta)<1$. The following theorem generalizes a result of Greechie and Miller [4].

THEOREM 1. Let $\alpha \in \Omega(\mathscr{A})$, where $\mathscr{A}$ is any premanual. Then $\alpha$ is pure if and only if $r(\alpha, \beta)=0$ holds for all $\beta \in \Omega(\mathscr{A})$ with $\beta \neq \alpha$.

Proof. Suppose first that $\alpha$ is pure, but that there exists $\beta \in \Omega(\mathscr{A})$ with $\beta \neq \alpha$ and $r(\alpha, \beta)>0$. Put $t=(1-r(\alpha, \beta))^{-1}$, noting that $1<t$. Put $\mu=t \alpha+(1-t) \beta$. If there existed $y \in X=\bigcup \mathscr{A}$ with $\mu(y)<0$, then we would have $0 \leqq \alpha(y)<\beta(y)$ and $\alpha(y) / \beta(y)<r(\alpha, \beta)$, a contradiction. It follows that $\mu(x) \geqq 0$ for all $x \in X$. If $E \in \mathscr{A}$, then $\sum_{x \in E} \mu(x)=1$, from which it follows that $\mu \in \Omega(\mathscr{A})$. Put $s=t^{-1}$, so that $0<s<1$ and $\alpha=s \mu+(1-s) \beta$. Since $\alpha$ is pure, we conclude that $\mu=\beta$, and hence that $\alpha=\beta$, a contradiction.

Conversely, suppose that $r(\alpha, \beta)=0$ for all $\beta \in \Omega(\mathscr{A})$ with $\beta \neq \alpha$, but that $\alpha$ is not pure. Then there exist $\mu, \beta \in \Omega(\mathscr{A})$ with $\mu \neq \beta$ and there exists a real number $s$ with $0<s<1$ such that $\alpha=s \mu+(1-s) \beta$. Evidently $\alpha \neq \beta$; hence there exists $y \in X$ with $\beta(y)>\alpha(y) \geqq 0$, $\alpha(y) / \beta(y)<1-s$. However, this gives the immediate contradiction $s \mu(y)<0$ and completes the proof.

3. Compound premanuals. Let $\mathscr{A}$ be a given premanual and $X=\bigcup \mathscr{A}$. Let $\Gamma=\Gamma(X)$ denote the free monoid (semigroup with unit 1) over the set $X$. An element of $\Gamma$ (other than the unit 1) is uniquely expressible in the form $x_{1} x_{2} \ldots x_{n}$ with $n$ a positive integer (called the length of the element) and $x_{1}, x_{2}, \ldots, x_{n} \in X$. We define the length of the unit 1 to be 0 and we denote the length of an element $a \in \Gamma$ by $|a|$. The elements of $\Gamma$ of length one are naturally identified with the corresponding elements of $X$, so that $X \subseteq \Gamma$.

A subset $A$ of $\Gamma$ is said to be bounded if there is a non-negative integer $n$ such that $|a| \leqq n$ for all $a \in A$. If $A$ and $B$ are subsets of $\Gamma$, we naturally define the product $A B$ by $A B=\{a b \mid a \in A, b \in B\}$. If $a \in \Gamma$ and $B \subseteq \Gamma$, we define $a B=\{a\} B$ and $B a=B\{a\}$.

If $E$ and $F$ are subsets of $\Gamma$ and if there exists, for each $e \in E, G_{e} \in \mathscr{A} \cup\{\{1\}\}$ such that $F=\bigcup_{e \in E} e G_{e}$, we shall say that $F$ is a direct successor of $E$. A set $\mathscr{H}$ of subsets of $\Gamma$ will be called an inductive class provided that it satisfies the following two conditions:

(i) $\{1\} \in \mathscr{H}$;

(ii) if $E \in \mathscr{H}$ and if $F$ is a direct successor of $E$, then $F \in \mathscr{H}$.

Notice that any $G \in \mathscr{A}$ is a direct successor of $\{1\}$; hence $\mathscr{A}$ is contained in any inductive class. The set of all nonempty subsets of $\Gamma$ is an inductive class, and the intersection of any family 
of inductive classes is again an inductive class. We shall denote by $\mathscr{A}^{c}$ the intersection of the family of all inductive classes of subsets of $\Gamma$, so that $\mathscr{A} \subseteq \mathscr{A}^{c}$ and $\mathscr{A}^{c}$ is the smallest inductive class of subsets of $\Gamma$. Since $\emptyset \notin \mathscr{A}^{c}, \mathscr{A}^{c}$ is a premanual called the compound premanual over $\mathscr{A}$.

Evidently, the collection of all bounded subsets of $\Gamma$ is an inductive class; hence every $E \in \mathscr{A}^{c}$ is bounded. A subset $K$ of $\Gamma$ is called an abridged set provided that, if $a, b \in K$ and if there exists $c \in \Gamma$ with $a=b c$, then $c=1$ (so that $a=b$ ). We shall now prove that every $E \in \mathscr{A}^{c}$ is an abridged set.

THEOREM 2. Let $\mathscr{A}$ be any premanual and let $E \in \mathscr{A}^{c}$. Then $E$ is an abridged set.

Proof. Let $X=\bigcup \mathscr{A}$ and let $\Gamma$ be the free monoid over $X$. Let $\mathscr{H}$ denote the set of all abridged subsets of $\Gamma$. It will suffice to prove that $\mathscr{H}$ is an inductive class. Clearly, $\{1\} \in \mathscr{H}$. Suppose that $E \in \mathscr{H}$ and that $F$ is a direct successor of $E$, but that $F \notin \mathscr{H}$. For each $e \in E$, there exists $G_{e} \in \mathscr{A} \cup\{\{1\}\}$ such that $F=\bigcup_{e \in E} e G_{e}$. Since $F \notin \mathscr{H}$, there exist $a, b \in F$ and $c \in \Gamma$, with $c \neq 1$ and $a=b c$. Since $a, b \in F$, there exist $d, e \in E, x \in G_{d}$ and $y \in G_{e}$ such that $a=d x$ and $b=e y$. Thus we have $d x=e y c$. Since $E$ is abridged, $x \neq 1$, for otherwise $d=e(y c)$, so that $y c=1, c=1$, a contradiction. Since $c \neq 1$, we can write $c=h z$ for some $h \in \Gamma, z \in X$. The equation $d x=e y h z$, together with the facts that $\Gamma$ is freely generated by $X$ and that $x, z \in X$, implies that $z=x$; hence we have $d=e y h$. Again, since $E$ is abridged, we must have $y h=1$; hence, $d=e, y=1$. Thus we have $e=e y=b$, and so $e \in F$. Also, $e x=d x=a \in F$; so $e x \in F$, with $x \in G_{e}, x \neq 1$. Since $G_{e} \in \mathscr{A} \cup\{\{1\}\}$ and $x \in G_{e}$ with $x \neq 1$, we have $1 \notin G_{e}$; hence $e \notin e G_{c}$. But, since $e \in F$, there must exist $k \in E$ with $e \in k G_{k}$. Hence $e=k w$ for some $w \in G_{k}$. Since $E$ is abridged, $w=1$ and $k=e$; hence $e \in e G_{e}$, a contradiction. The proof is complete.

Corollary 3. Let $\mathscr{A}$ be a premanual and let $E \in \mathscr{A}^{c}$. For each $e \in E$, let $G_{e} \in \mathscr{A} \cup\{\{1\}\}$. Then, if $d, e \in E$ with $d \neq e$, it follows that $d G_{d} \cap e G_{e}=\emptyset$.

THEOREM 4. Let $\mathscr{A}$ be a premanual with $X=\bigcup \mathscr{A}$ and let $\Gamma$ be the free monoid over $X$. Then $\bigcup_{\mathscr{A}}^{c}=\Gamma$.

Proof. It will suffice to show that each element $a \in \Gamma$ belongs to at least one set $E \in \mathscr{A}^{c}$. We prove this by induction on $|a|$. If $|a|=0$, then $a=1 \in\{1\} \in \mathscr{A}^{c}$. Suppose that the asser:ion is true for all $a \in \Gamma$ with $|a|=n$. Let $b \in \Gamma$ with $|b|=n+1$. Then we can write $b=a x$ with $|a|=n$ and $x \in X$. By hypothesis, there exists $E \in \mathscr{A}^{c}$ with $a \in E$. Since $x \in X$, there exists $G \in \mathscr{A}$ with $x \in G$. For each $e \in E$, define $G_{e}=G$, and note that $F=\bigcup_{e \in E} e G_{e} \in \mathscr{A}^{c}$, since $\mathscr{A}^{c}$ is an inductive class. Since $b=a x \in a G_{a} \subseteq F$, the proof is complete.

4. Weight functions for compound premanuals. For the remainder of this paper, we assume that $\mathscr{A}$ is a premanual with $\Omega(\mathscr{A}) \neq \emptyset$ and we put $X=\bigcup \mathscr{A}$. We also denote by $\Gamma$ the free monoid over $X$. By a transition function for the premanual $\mathscr{A}^{c}$ we mean a function $f: \Gamma \times X \rightarrow \mathbb{R}$ such that, for every $e \in \Gamma, f(e, \cdot) \in \Omega(\mathscr{A})$. Thus, a transition function can be regarded as a family of weight functions for $\mathscr{A}$ indexed by the elements of $\Gamma$. If $f$ is a transition function for $\mathscr{A}^{c}$, we define a real-valued function $\omega_{f}$ on $\Gamma$ by recursion as follows:

(1) $\omega_{f}(1)=1$;

(2) if $a \in \Gamma$ and $x \in X$, then $\omega_{f}(a x)=\omega_{f}(a) f(a, x)$. 
In particular, we have $\omega_{f}(x)=f(1, x)$ for all $x \in X$. For $x_{1}, x_{2}, \ldots, x_{n} \in X, n \geqq 2$, we will then have

$$
\omega_{f}\left(x_{1} x_{2} \ldots x_{n}\right)=f\left(1, x_{1}\right) \prod_{j=2}^{n} f\left(x_{1} x_{2} \ldots x_{j-1}, x_{j}\right) .
$$

THEOREM 5. If $f$ is any transition function for $\mathscr{A}^{c}$, then $\omega_{f} \in \Omega\left(\mathscr{A}^{c}\right)$.

Proof. Evidently, $\omega_{f}(a) \geqq 0$ for all $a \in \Gamma$. Thus it will suffice to show that, for any $E \in \mathscr{A}^{c}, \sum_{e \in E} \omega_{f}(e)=1$. Thus, let $\mathscr{H}$ denote the set of all sets $E \in \mathscr{A}^{c}$ such that $\sum_{e \in E} \omega_{f}(e)=1$. It will be enough to show that $\mathscr{H}$ is an inductive class. Clearly, $\{1\} \in \mathscr{H}$. Thus, let $E \in \mathscr{H}$, and suppose that $F$ is a direct successor of $E$. Then, for every $e \in E$, there exists $G_{e} \in \mathscr{A} \cup\{\{1\}\}$ such that $F=\bigcup_{e \in E} e G_{e}$. By Corollary 3, the latter is a disjoint union. Let us temporarily fix an $e \in E$ and put $G=G_{e}$. If $G=\{1\}$, then $\sum_{a \in e G} \omega_{f}(a)=\omega_{f}(e)$. On the other hand, if $G \neq\{1\}$, then $G \in \mathscr{A}$ and we have

$$
\sum_{a \in \in G} \omega_{f}(a)=\sum_{x \in G} \omega_{f}(e x)=\sum_{x \in G} \omega_{f}(e) f(e, x)=\omega_{f}(e) \sum_{x \in G} f(e, x)=\omega_{f}(e) .
$$

It follows that $\sum_{a \in F} \omega_{f}(a)=\sum_{e \in E} \omega_{f}(e)=1$; hence $\mathscr{H}$ is an inductive class and the proof is complete.

Lemma 6. Let $\omega \in \Omega\left(\mathscr{A}^{c}\right)$ and let $a \in \Gamma, G \in \mathscr{A}$. Then $\sum_{x \in \mathbf{G}} \omega(a x)=\omega(a)$.

Proof. By Theorem 4, there exists $E \in \mathscr{A}^{c}$ with $a \in E$. For $e \in E$ with $e \neq a$, define $G_{e}=\{1\}$. Define $G_{a}=G$. Put $F=\bigcup_{e \in E} e G_{e}$, noting that $F \in \mathscr{A}^{c}$. Put $H=E \mid a$. We now have

$$
1=\sum_{b \in \boldsymbol{F}} \omega(b)=\sum_{e \in \boldsymbol{H}} \omega(e)+\sum_{x \in G} \omega(a x)=1-\omega(a)+\sum_{x \in G} \omega(a x),
$$

and the lemma is proved.

Suppose that $f$ is a transition function for $\mathscr{A}^{c}$ and that $d$ belongs to $\Gamma$. We then define a new transition function $f / d$, called $f$ conditioned by $d$, by the following prescription:

$$
(f / d)(a, x)=\left\{\begin{array}{lll}
f(d, x) & \text { if } \quad \omega_{f}(d a)=0 \\
f(d a, x) & \text { if } \quad \omega_{f}(d a) \neq 0
\end{array}\right.
$$

for $a \in \Gamma, x \in X$.

THEOREM 7. Let $f$ be a transition function for $\mathscr{A}^{c}$ and let $d \in \Gamma$. Put $g=f / d$. Then, for any $a \in \Gamma$, we have $\omega_{g}(a) \omega_{f}(d)=\omega_{f}(d a)$.

Proof. The proof is by induction on $|a|$. If $|a|=0$, then $a=1$ and the result is evidently true. Suppose that the result holds for $|a|=n$ and let $b \in \Gamma$ with $|b|=n+1$. Then there exists $a \in \Gamma$ and $x \in X$, with $|a|=n, b=a x$. By hypothesis, $\omega_{g}(a) \omega_{f}(d)=\omega_{f}(d a)$. Hence $\omega_{g}(b) \omega_{f}(d)=\omega_{g}(a x) \omega_{f}(d)=\omega_{g}(a) g(a, x) \omega_{f}(d)=\omega_{f}(d a) g(a, x)$. Hence, if $\omega_{f}(d a) \neq 0$, we have $\omega_{g}(b) \omega_{f}(d)=\omega_{f}(d a) f(d a, x)=\omega_{f}(d a x)=\omega_{f}(d b)$ as desired. Thus we can suppose that 
$\omega_{f}(d a)=0$. This gives $\omega_{\theta}(b) \omega_{f}(d)=0$, and we are obliged to prove that $\omega_{f}(d b)=0$. Since $x \in X$, there exists $G \in \mathscr{A}$ with $x \in G$. By Lemma 6 ,

$$
0=\omega_{f}(d a)=\sum_{y \in G} \omega_{f}(d a y) \geqq \omega_{f}(d a x)=\omega_{f}(d b) \geqq 0 ;
$$

hence $\omega_{f}(d b)=0$ as desired.

A transition function $f$ for $\mathscr{A}^{c}$ is said to be normalized if it satisfies the following condition: For all $a \in \Gamma$ and all $x \in X$, if $\omega_{f}(a)=0$, then $f(a, x)=f(1, x)$. Suppose that $\alpha \in \Omega\left(\mathscr{A}^{c}\right)$ and define $f: \Gamma \times X \rightarrow \mathbb{R}$ as follows. For $a \in \Gamma$ and $x \in X$,

$$
f(a, x)=\left\{\begin{array}{lll}
\alpha(x) & \text { if } & \alpha(a)=0, \\
\frac{\alpha(a x)}{\alpha(a)} & \text { if } & \alpha(a) \neq 0 .
\end{array}\right.
$$

As a consequence of Lemma 6, we see that $f$ is a transition function for $\mathscr{A}^{c}$, and direct calculation reveals that $\omega_{f}=\alpha$, from which it easily follows that $f$ is normalized. A final calculation shows that, if $g$ is any normalized transition function for $\mathscr{A}^{c}$ such that $\omega_{g}=\alpha$, then $g=f$. Thus we have the following lemma.

LEMMA 8. The mapping $f \mapsto \omega_{\text {s }}$ provides a one-to-one correspondence between normalized transition functions $f$ for $\mathscr{A}^{c}$ and the set $\Omega\left(\mathscr{A}^{c}\right)$ of all weight functions $\omega_{f}$ for $\mathscr{A}^{c}$.

Suppose that $\alpha \in \Omega\left(\mathscr{A}^{c}\right)$ and that $d \in \Gamma$. Let $f$ be the unique normalized transition function for $\mathscr{A}^{c}$ such that $\omega_{f}=\alpha$. We can now form the conditioned transition function $f / d$ and thence the weight function $\omega_{s / d}$. We call $\omega_{s / d}$ the weight function obtained by conditioning $\alpha$ by $d$ and we introduce the notation $\alpha / d$ for $\omega_{s / d}$. According to Theorem 7 , we have the identity $(\alpha / d)(a) \cdot \alpha(d)=\alpha(d a)$ for all $a \in \Gamma$. In particular, if $\alpha(d) \neq 0$, we have

$$
(\alpha / d)(a)=\frac{\alpha(d a)}{\alpha(d)},
$$

a formula which is analogous to the classical definition of conditional probability. An easy calculation shows that the transition function $f / d$ is normalized, so that $f / d$ is the unique normalized transition function corresponding to $\alpha / d$ according to Lemma 8 .

Continuing with the above notation, we notice that from the equation $\alpha(d a)=(\alpha / d)(a) \cdot \alpha(d)$ we can deduce that, if $\alpha(d)=0$, then $\alpha(d a)=0$ holds for all $a \in \Gamma$. From this we see that, if $\alpha(d)=0$, then we have

$$
(\alpha / d)\left(x_{1} x_{2} \ldots x_{n}\right)=\alpha\left(x_{1}\right) \alpha\left(x_{2}\right) \ldots \alpha\left(x_{n}\right),
$$

for $x_{1}, x_{2}, \ldots, x_{n} \in X, n \geqq 1$. This suggests a slight extension of the above notation. Given any normalized transition function $f$ for $\mathscr{A}^{c}$, we define a transition function $f / *$ for $\mathscr{A}^{c}$ by $(f / *)(a, x)=f(1, x)$ for all $a \in \Gamma$ and all $x \in X$. Evidently, $f / *$ is normalized. Given any weight function $\alpha \in \Omega\left(\mathscr{A}^{c}\right)$, we now define $\alpha / *$ as follows. Let $f$ be the unique normalized transition function for which $\alpha=\omega_{f}$, and define $\alpha / *=\omega_{f / *}$. Evidently,

$$
(\alpha / *)\left(x_{1} x_{2} \ldots x_{n}\right)=\alpha\left(x_{1}\right) \alpha\left(x_{2}\right) \ldots \alpha\left(x_{n}\right)
$$


holds for $x_{1}, x_{2}, \ldots, x_{n} \in X, n \geqq 1$. In particular, $(\alpha / *)(a b)=(\alpha / *)(a) \cdot(\alpha / *)(b)$ holds for all $a, b \in \Gamma$ and we have the result that, if $d \in \Gamma$ with $\alpha(d)=0$, then $\alpha / d=\alpha / *$.

5. The stability of pure weights under conditioning. In the present section, we shall prove the main theorem of this paper, namely that, if $\alpha$ belongs to $\Omega_{p}\left(\mathscr{A}^{c}\right)$ and if $d$ is any element of $\Gamma$, then the conditioned weight function $\alpha / d$ also belongs to $\Omega_{p}\left(\mathscr{A}^{c}\right)$.

LemMa 9. Let $\alpha \in \Omega_{p}\left(\mathscr{A}^{c}\right)$ and let $d \in \Gamma$ with $\alpha(d) \neq 0$. Define a real-valued function $\beta$ on $X=\bigcup \mathscr{A}$ by $\beta(x)=\alpha(d x) / \alpha(d)$ for all $x \in X$. Then $\beta \in \Omega_{p}(\mathscr{A})$.

Proof. Suppose that $\beta \notin \Omega_{p}(\mathscr{A})$. By Theorem 1, there exists $\mu \in \Omega(\mathscr{A})$, with $\mu \neq \beta$ and $r(\beta, \mu)>0$. Let $f$ be the unique normalized transition function for $\mathscr{A}^{c}$ such that $\alpha=\omega_{f}$. Define a transition function $g$ for $\mathscr{A}^{c}$ as follows.

for $a \in \Gamma, x \in X$.

$$
g(a, x)=\left\{\begin{array}{lll}
f(a, x) & \text { if } & a \neq d \\
\mu(x) & \text { if } & a=d
\end{array}\right.
$$

Suppose that $\omega_{g}=\alpha$. Then, for any $x \in X, \alpha(d) f(d, x)=\omega_{f}(d) f(d, x)=\omega_{f}(d x)=\alpha(d x)=$ $\omega_{\theta}(d x)=\omega_{g}(d) g(d, x)=\alpha(d) \mu(x)$; hence, since $\alpha(d) \neq 0, f(d, x)=\mu(x)$ holds for all $x \in X$. However, since $\alpha(d) \neq 0, f(d, x)=\beta(x)$ holds for all $x \in X$, and we obtain the contradiction $\mu=\beta$. Thus $\omega_{\theta} \neq \alpha$.

Since $\alpha \in \Omega_{p}\left(\mathscr{A}^{c}\right)$ and $\omega_{g} \in \Omega\left(\mathscr{A}^{c}\right)$ with $\alpha \neq \omega_{g}$, Theorem 1 gives $r\left(\alpha, \omega_{g}\right)=0$. It follows that there exists an element $c \in \Gamma$ with $0 \leqq \alpha(c)<\omega_{g}(c), \alpha(c) / \omega_{g}(c)<r(\beta, \mu)$. Evidently, $c \neq 1$; hence we can write $c=x_{1} x_{2} \ldots x_{n}$ with $n \geqq 1$ and $x_{1}, x_{2}, \ldots, x_{n} \in X$. For $1 \leqq j \leqq n$, define $c_{j}=1$ if $j=1$ and $c_{j}=x_{1} x_{2} \ldots x_{j-1}$ if $2 \leqq j \leqq n$. We have

and

$$
\alpha(c)=f\left(c_{1}, x_{1}\right) f\left(c_{2}, x_{2}\right) \ldots f\left(c_{n}, x_{n}\right)
$$

$$
\omega_{g}(c)=g\left(c_{1}, x_{1}\right) g\left(c_{2}, x_{2}\right) \ldots g\left(c_{n}, x_{n}\right)
$$

Since $\alpha(c) \neq \omega_{g}(c)$, there must exist a positive integer $i$ with $1 \leqq i \leqq n$ and $f\left(c_{i}, x_{i}\right) \neq g\left(c_{i}, x_{i}\right)$. From the definition of $g\left(c_{i}, x_{i}\right)$ it follows that $c_{i}=d$ and $g\left(c_{i}, x_{i}\right)=\mu\left(x_{i}\right)$. We also have $f\left(c_{i}, x_{i}\right)=f\left(d, x_{i}\right)=\beta\left(x_{i}\right)$, since $\alpha(d) \neq 0$. For $1 \leqq j \leqq n$ with $j \neq i$, we have $c_{j} \neq c_{i}=d$; hence $g\left(c_{j}, x_{j}\right)=f\left(c_{j}, x_{j}\right)$. From the condition $0 \leqq \alpha(c)<\omega_{g}(c)$ we deduce that $g\left(c_{j}, x_{j}\right)>0$ for $1 \leqq j \leqq n$ and that

$$
\frac{\beta\left(x_{i}\right)}{\mu\left(x_{i}\right)}=\frac{\alpha(c)}{\omega_{g}(c)}<r(\beta, \mu),
$$

an immediate contradiction.

COROLlARY 10. Let $\alpha \in \Omega_{p}\left(\mathscr{A}^{c}\right)$ and let $f$ be the unique normalized transition function for $\mathscr{A}^{c}$ for which $\omega_{f}=\alpha$. Then, for every element $a \in \Gamma, f(a, \cdot) \in \Omega_{p}(\mathscr{A})$.

Proof. If $\alpha(a) \neq 0$, then $f(a, x)=\alpha(a x) / \alpha(a)$ for all $x \in X$; so $f(a, \cdot) \in \Omega_{p}(\mathscr{A})$, by Lemma 9 . On the other hand, if $\alpha(a)=0$, then $f(a, x)=f(1, x)=\alpha(x)=\alpha(1 x) / \alpha(1)$ for all $x \in X$, so that, again by Lemma $9, f\left(a,,^{\circ}\right) \in \Omega_{p}(\mathscr{A})$, and the corollary is proved.

A normalized transition function $f$ for $\mathscr{A}^{c}$ will be called pure if $f\left(a,{ }^{\cdot}\right) \in \Omega_{p}(\mathscr{A})$ holds for 
every $a \in \Gamma$. Corollary 10 says that, if $\omega_{f}$ is a pure weight function for $\mathscr{A}^{c}$, then $f$ is pure. In the following theorem we shall establish the converse.

THEOREM 11. Let $f$ be a normalized transition function for $\mathscr{A}^{c}$. Then $f$ is pure if and only if $\omega_{f} \in \Omega_{p}\left(\mathscr{A}^{c}\right)$.

Proof. We know already that, if $\omega_{f}$ is pure, then so is $f$. Suppose, then, that $f$ is pure, but that $\omega_{f}$ is not pure. Then, by Theorem 1, there exists a normalized transition function $g \neq f$ such that $0<r\left(\omega_{f}, \omega_{g}\right)$. Suppose that $f(1, \cdot) \neq g(1, \cdot)$. Since $f(1, \cdot) \in \Omega_{p}(\mathscr{A})$, Theorem 1 gives $r(f(1, \cdot), g(1, \cdot))=0$; hence there exists $x \in X$ such that $0 \leqq f(1, x)<g(1, x)$ and $f(1, x) / g(1, x)<r\left(\omega_{f}, \omega_{\theta}\right)$. Since $\omega_{f}(x)=f(1, x)$ and $\omega_{g}(x)=g(1, x)$, the latter inequality cannot be true; hence we conclude that $f(1, \cdot)=g(1, \cdot)$.

Choose $b \in \Gamma$ with $|b|$ minimal such that $f(b, \cdot) \neq g(b, \cdot)$. Since $b \neq 1$, we can write $b=x_{1} x_{2} \ldots x_{n}$ with $n \geqq 1$ and $x_{1}, x_{2}, \ldots, x_{n} \in X$. Put $c_{1}=1$ and $c_{j}=x_{1} x_{2} \ldots x_{j-1}$ for $2 \leqq j \leqq n$. We have

$$
\omega_{f}(b)=\prod_{j=1}^{n} f\left(c_{j}, x_{j}\right)=\prod_{j=1}^{n} g\left(c_{j}, x_{j}\right)=\omega_{g}(b),
$$

since $\left|c_{j}\right|<n=|b|$ for all $j=1,2, \ldots, n$. Suppose that $\omega_{f}(b)=\omega_{g}(b)=0$. Then, since $f$ and $g$ are normalized,

$$
f(b, \cdot)=f(1, \cdot)=g(1, \cdot)=g(b, \cdot)
$$

a contradiction. We conclude that $\omega_{f}(b)=\omega_{g}(b) \neq 0$.

Since $f(b, \cdot) \neq g(b, \cdot)$ and $f(b, \cdot) \in \Omega_{p}(\mathscr{A})$, then, by Theorem $1, r(f(b, \cdot), g(b, \cdot))=0$. Hence there exists $x \in X$ such that

$$
\frac{f(b, x)}{g(b, x)}<r\left(\omega_{f}, \omega_{g}\right)<\frac{\omega_{f}(b x)}{\omega_{g}(b x)}=\frac{\omega_{f}(b) f(b, x)}{\omega_{g}(b) g(b, x)},
$$

yielding the contradiction that $f(b, x) / g(b, x)$ is less than itself. This contradiction proves the theorem.

THEOREM 12. Let $\alpha \in \Omega_{p}\left(\mathscr{A}^{c}\right)$ and let $d \in \Gamma$. Then the conditioned weight function $\alpha / d$, as well as $\alpha / *$, belong to $\Omega_{p}\left(\mathscr{A}^{c}\right)$.

Proof. Let $f$ be the unique normalized transition function for $\mathscr{A}^{c}$ such that $\omega_{f}=\alpha$. By Theorem $11, f$ is pure. From the definitions of $f / d$ and $f / *$, we see immediately that $f / d$ and $f / *$ are pure; hence, by Theorem $11, \alpha / d=\omega_{f / d} \in \Omega_{p}\left(\mathscr{A}^{c}\right)$ and $\alpha / *=\omega_{f / *} \in \Omega_{p}\left(\mathscr{A}^{c}\right)$ as desired.

6. Concluding remarks. There are many known examples of " conditioning" processes which preserve the "purity" of "stochastic models". Indeed, the classical example is obtained as follows: Let $\mathscr{B}$ denote any Boolean algebra. From $\mathscr{B}$, we construct a premanual $\mathscr{A}=\left\{E \subseteq \mathscr{B} \mid E\right.$ is a finite set of pairwise disjoint nonzero elements of $\mathscr{B}$ and $\left.\sum_{e \in E} e=1\right\}$. Evidently, the weight functions in $\Omega(\mathscr{A})$ are in a natural one-to-one correspondence with the finitely additive probability measures on $\mathscr{B}$. We shall identify a weight function $\omega \in \Omega(\mathscr{A})$ with the corresponding probability measure. The pure weights now correspond to the points in the Stone space affiliated with $\mathscr{B}$. Suppose that $\omega \in \Omega(\mathscr{A})$ and that $a \in \mathscr{B}$ with $\omega(a) \neq 0$. By 
" conditioning" $\omega$ by $a$, we can define a new weight function $\omega_{a} \in \Omega(\mathscr{A})$ by $\omega_{a}(b)=\omega(a b) / \omega(a)$ for all $b \in \mathscr{B}$. It is easy to see that, if $\omega$ is pure, so is $\omega_{a}$.

A second example arises in conventional non-relativistic quantum mechanics. To construct this example, let $\mathscr{H}$ be a complex, separable, infinite-dimensional Hilbert space and let $\mathscr{D}$ be the set of all von Neumann density operators on $\mathscr{H}$. Let the premanual $\mathscr{A}$ consist of all countable sets $\left\{P_{1}, P_{2}, \ldots\right\}$ of pairwise orthogonal nonzero projection operators on $\mathscr{H}$ such that $\sum_{i} P_{i}=1$. For each $D \in \mathscr{D}$, define the weight function $\omega_{D}$ by $\omega_{D}(P)=\operatorname{Tr}(D P)$ for all nonzero projection operators $P$ on $\mathscr{H}$. These weight functions are now in a natural one-toone correspondence with the quantum mechanical states. Furthermore, the weight function $\omega_{D}$ corresponds to a pure state if and only if $D=D^{2}$. Evidently, $\omega_{D}$ is a pure weight if and only if it corresponds to a pure quantum mechanical state. Suppose that $D \in \mathscr{D}$ and that $P$ is a projection operator on $\mathscr{H}$ such that $\operatorname{Tr}(D P) \neq 0$. The usual quantum mechanical " conditioning by $P$ " $\left[6\right.$, p. 333; 5] converts $D$ into $D_{P}=(\operatorname{Tr}(D P))^{-1} P D P$. It is easy to check that this conditioning preserves pure weights.

In Pool's work on the logic of quantum mechanics, it is shown that (under suitable hypotheses on the event-state-operation structures under consideration) pure states are stable under conditioning by operations precisely when the quantum logic is semimodular [7].

Our Theorem 12 provides still another example of the " stability of purity under conditioning". However, it can be shown that, if the weight functions on a compound premanual are conditioned not by outcomes, but by so-called " events", the purity of the weight functions is not generally preserved.

\section{REFERENCES}

1. D. Foulis and C. Randall, Operational statistics I, basic concepts, J. Mathematical Physics 13 (1972), 1667-1675.

2. D. Foulis and C. Randall, Conditioning maps on orthomodular lattices, Glasgow Math. J. 12 (1971), 35-42. 119-132.

3. R. Greechie, Orthomodular lattices admitting no states, J. Combinatorial Theory 10 (1971),

4. R. Greechie and F. Miller, On structures related to states on an empirical logic: I. Weights on finite spaces, Kansas State University mimeographed notes, 1969.

5. G. Lüders, Über die Zustandsänderung durch den Messprozess, Ann. Physik 8 (1951), 322-328.

6. A. Messiah, Quantum Mechanics. Vol. 1 (Amsterdam, 1961). 212-228.

7. J. Pool, Semimodularity and the logic of quantum mechanics, Comm. Math. Phys. 9 (1968), 363-374.

8. C. Randall and D. Foulis, An approach to empirical logic, Amer. Math. Monthly 77 (1970),

9. C. Randall and D. Foulis, States and the free orthogonality monoid, Math. Systems Theory 6 (1972), 268-276.

\section{UNIVERSITY OF MASSACHUSETTS}

AMHERST, MASS. 01002 